\title{
Mechanistic explanation in Physics
}

\section{Laura Felline}

fellinelaura [at] gmail [dot] com

\section{Introduction}

The idea at the core of the New Mechanical account of explanation can be summarized in the claim that explaining means showing 'how things work'. This simple motto hints at three basic features of Mechanistic Explanation (ME): ME is an explanation-how, that implies the description of the processes underlying the phenomenon to be explained and of the entities that engage in such processes. These three elements trace a fundamental contrast with the view inherited from Hume and later from strict logical empiricism (see Creath 2017), focused on epistemic and formal features of science and according to which issues concerning the kind of entities and processes that lie within a theory's domain are extraneous to science and belong instead to ontology or metaphysics. Philosophers belonging to the new mechanical philosophy believe that the received view of scientific explanation (Hempel 2001), pivoting on the notion of law of nature, ${ }^{1}$ overshadows this insight.

Since its origin in the $17^{\text {th }}$ century, mechanical philosophy aimed to explain natural phenomena by reducing them to mechanisms. Traditional attempts to define the concept of mechanism involved the identification of a limited set of fundamental elements as, for instance, contact action, action at a distance, inertial motion (see e.g. Hesse 2005), and, more recently, transmission of a mark, or of a conserved quantity (see Frisch, this volume). The new mechanical philosophy rejects this austere characterization of mechanisms and mechanistic explanation and aim at providing a novel, philosophically rigorous explication of the concept of mechanism and of its role in scientific explanation and practice.

ME has been adopted with profit in philosophy of special sciences (for instance in biomedical sciences, e.g. in the explanation of chemical transmission at synapses ((Machamer, Darden and Craver 2000), MDC henceforth); but also in social sciences, e.g. the three kinds of social mechanisms in Coleman's analysis of Max Weber's account of the role of the Protestant ethic in the growth of capitalism (Hedström and Swedberg 1998)), where exceptionless regularities are rarely ever found. In physics, it is generally possible to formulate explanations in law-based form, with the result that the plurality of explanatory forms might be overlooked. This should not come as a surprise, given that physics was the main inspiration for logical empiricists, and, in particular, Newtonian physics

Unless otherwise specified, in this paper we indicate with the expression 'law of nature' the non-metaphysically charged notion of exceptionless regularities that support counterfactuals. See Lange (this volume) for a variety of accounts of laws of nature. 
was a template for Hempel's formulation of the covering law model. However, this situation is unfortunate, since, we will argue, knowing how things work is often part of the explanation of physical phenomena. In this chapter, we provide an introduction to the basic features of ME, with specific focus on its application to physics (section 1). The main part of the chapter is devoted to the defence of two theses: on the one hand, some domains of physics are not compatible with mechanistic reasoning and explanation (section 2); on the other hand, a comprehensive account of explanation in physics can't dispense with ME (section 3).

\section{Mechanistic Explanation: general features}

A ME of a phenomenon $P$ requires the description, which may be more or less idealized, of the mechanisms underlying the occurrence of $P$. The novelty of the new mechanical philosophy with respect to other accounts of mechanism (e.g. Salmon's (1984), see also (Frisch this volume)) lies in its novel definition of mechanism as organized entities and activities. The literature displays many variations over this basic idea, here we report a minimal characterization of the concept: ${ }^{2}$

"A mechanism for a phenomenon consists of entities (or parts) whose activities and interactions are organized in such a way that they produce the phenomenon". (Glennan 2017, Ch. 2)."

A familiar example might serve as an illustration. The pressure of a gas in equilibrium inside a piston at constant temperature increases when its volume decreases and vice versa. A ME of this phenomenon displays a kinetic model of the ideal gas, described as a mechanism composed of Newtonian atoms and molecules (entities), with constant, rapid and random motion (activities) and perfectly elastic collisions (interactions). The gas' pressure corresponds to the force resulting from the totality of the particles' hits on the wall of the container. The smaller is the volume occupied by the gas, the higher is the number of particles per unit of volume and the more frequent are the hits to the wall of the container. It follows that a reduction of volume corresponds to an increase of pressure.

Mechanisms are organized in nested hierarchies, in the sense that a mechanism's components are themselves mechanisms and their behaviour is therefore mechanistically explainable through description of its components and their activities (Glennan 2011; MDC, 5.1). However, a theory describing such a tiered structure of mechanistic composition, always bottoms-out with a stable set of mechanisms that are fundamental in that theory, in the sense that the behaviour of the entities that compose such mechanisms provide the basic building blocks of MEs in said domains and cannot be explained within the theory itself. Bottoming-out is domain-specific: while the behaviour of entities cannot be explained within a theory where they are fundamental, they might be explainable by the mechanisms of a lower-level theory. For instance, a gas in a piston is an elementary mechanism in

2 Other definitions can be found e.g. in (MDC, 3), (Glennan 2002, S344), (Bechtel and Abrahamsen 2005) and (Illari and Williamson 2012). 
classical thermodynamics; its behaviour is not explainable within such a theory, but it can be explained by the lower-level mechanistic models displayed by the kinetic theory of gases. ${ }^{3}$ As another example, in fluid dynamics a fluid is a fundamental entity whose lack of resistance to deformation under the action of a force is unexplained. However, the lack of resistance to deformation is explained by the lower-level mechanistic decomposition: fluids are entities composed of particles whose bonding force is so weak, that it opposes a negligible resistance to any force cutting particles apart therefore the lack of resistance to deformation.

Bottoming-out raises the ontological issue whether and when the structure of nested hierarchy ends. It is currently matter of debate whether an ontologically fundamental mechanistic level can exist or whether there are mechanisms "all the way down". According to Glennan (2017, ch. 5.5), this is an empirical question and the possibility of mechanisms "all the way down" cannot be ruled out a priori.

The formulation of a ME requires in general the isolation of the entities that are the components of the mechanism (structural decomposition) and of the activities in which entities engage (functional decomposition). In a satisfactory ME, these two elements must be adequately integrated (Bechtel and Richardson 2010).

A mechanism is always a mechanism for a behaviour. The same mechanical system may exhibit different behaviours, and which structural and functional decompositions are correct for an explanation depends on the behaviour to be explained (see Craver 2013).

Another novelty of the new ME with respect to its predecessors is the rejection of explanatory fundamentalism, i.e. the assumption that the best explanation is provided in terms of the most fundamental theories (Craver 2007, p. 11, n. 13). Crucially, the new mechanical philosophy imposes no a priori constraint (other than basic standards of scientificity, e.g. coherence, empirical adequacy, predictive power or salience) to the features of such building blocks, which vary when the available fundamental mechanisms of a given domain are found insufficient to explain new discovered phenomena. For instance, consider the development of classical mechanical philosophy, ${ }^{4}$ based on the assumption that matter is the sole physical entity and motion and transmission of impulse by contact are the fundamental activities (Kochiras 2013). As new phenomena were discovered in optics, electricity and magnetism, the explanatory power of this model turned out to be insufficient, so that corpuscles as centres of force capable of attracting and repelling at a distance were added to the stable set of fundamental entities and activities. Finally, Maxwell's theory established that electrical and magnetic phenomena could not be explained exclusively in terms of a theory of action (although at a distance) between bodies, but required a new kind of fundamental active physical entity: the

3 See section 7 of this volume for further discussion of these explanations.

4 See section 1 for detailed discussion of classical mechanics. 
electromagnetic field. ${ }^{5}$

Entities interact in virtue of their properties, and interactions are the carriers of change and production. There is general consensus that there is no ME without change and production, which makes ME a productive account of causal explanation, as opposed to a relevance account (Hall 2004, Glennan 2011).

The third constitutive element of a mechanism, together with entities and activities, is organization: a set of entities and their activities does not constitute a mechanism, if they are not arranged in the correct way. Spatio-temporal relations constitute the most common example of organization of mechanisms part: in order for one idle wheel to transmit torque between two others, it is not sufficient that the wheels' teeth have the right size, but they must be spatially arranged in such a way that the teeth of the middle wheel fit together with the indentation of the others. Another example of organizational feature is the kind of geometry (e.g. Euclidean or Minkowskian) instantiated by the spatiotemporal properties of a mechanism.

\section{Limits of Mechanistic Explanation in physics}

As the familiar examples illustrated so far show, mechanisms can be found all over physics. ${ }^{6}$ In this section we are going to show that ME is inapplicable to some explanations in physics.

A significant limit for the mechanistic account is represented by the class of explanations that are independent of the micro-constitution or micro-dynamics of the systems displaying the behaviour to be explained. Notable examples in this class are explanations based on conservation laws (for example the explanation of Archimedes' Principle, exploiting the conservation of energy (Lange 2011)) and the universal behaviour of systems near their critical points (so-called renormalization group (RG) explanations (Batterman 2002) $)^{7}$ ). However, what conclusion should be drawn from this limit is matter of debate. Kuhlmann (2011), for instance, argues that RG explanations are actually MEs, therefore the fact that the new mechanistic account does not cover them shows that it is too restrictive. He therefore proposes a suitable modification of the account to include the class of what he calls 'structural mechanisms'.

Notice that, even granted that such explanations are not MEs, yet individual instances of the explanandum behaviour taken under consideration are mechanistically explained in physics. For instance, the RG explanation of why, when near their critical points, fluids of different molecular constitution behave in a similar way, might be non-mechanistic, but the explanation of the behaviour of an individual fluid near its critical point, is. This fact traces a significant difference between said

5 For an overview of the development of the concept of field, see Mary Hesse's magisterial book "Forces and Fields" (2005).

6 See also (Kuhlmann 2017) for other examples.

7 See Batterman (this volume) for more on universality. 
non-ME and others that, as well as being independent of the micro constitution or micro dynamics of the systems displaying the behaviour to be explained, are also understood as accounting for phenomena that are not even in principle mechanically explainable.

As an example, take the geometrical explanation of length contraction in Special Relativity (SR). ${ }^{8}$ Three features separate this explanation from ME (Felline 2015). The first two features are the abstraction and generality of the model: since in this explanation no microphysical or dynamical element is relevant, its interpretation as ME would define the model of a 'universal' mechanism, implemented by every physical system irrespectively of its features - even of whether or not they are complex systems. Including such a kind of explanation, Felline argues, would imply a trivialization of the concept of mechanism, and of ME. The third feature is a consequence of the first two. Janssen (2013) argues that the universality of SR's explanations justifies a kinematical interpretation of relativistic effects. Since (at least in its 'orthodox' interpretation) ${ }^{9}$ length contraction is not causally produced, therefore a fortiori it does not represent the behaviour of a mechanism. On the other hand, length contraction and time dilation are the instantiations of a feature of the spatio-temporal organization of any complex system relativistically described. Non-MEs of this kind are the so-called 'structural explanations' and might include explanations in quantum theory, as for instance the explanations of non-locality (Dorato and Felline 2011) and of the uncertainty relations (Felline 2015).

According to Kuhlmann and Glennan (2014), the vast majority of quantum phenomena, even when they depend on the underlying dynamical and constitutive details of the systems involved, is non-mechanistically explainable, for at least three reasons. First of all, entities in a mechanism interact in virtue of their properties, but quantum states do not associate determinate values to every property of a system. For instance, quantum states associated with a determinate spin value in the $\mathrm{x}$ axis, are not associated with a determinate spin value in the y axis, and vice versa. Following the standard interpretation of QT, such indeterminacy is ontological: quantum systems in a state with determinate spin value in the $\mathrm{x}$ axis do not possess a determinate spin in the $\mathrm{y}$ axis. According to Kuhlmann and Glennan, this situation is not compatible with ME: first of all, they argue, mechanisms are composed of objects with definite properties; secondly, components of a mechanism are interconnected via local interactions, therefore spatio-temporal localization is an especially important property for mechanisms, so the indeterminateness of position is especially problematic for ME; third, entanglement ${ }^{10}$ seems to undermine the possibility of decomposition into separate parts. However, Kuhlmann's and Glennan's conclusion can be contrasted on the basis of the arguably too restrictive characterization of mechanism they provide. For instance, as the two philosophers anticipate, one

\footnotetext{
See Maudlin, this volume.

9 Though see Brown and Read (this volume) for discussion of dissenting views.

10 See Healey (this volume).
} 
might reject the assumption of localizability (Bechtel and Richardson 2010). Also, the problem with entanglement seems to have origin in the requirement that "parts have properties that are relatively stable over time and that at least theoretically these parts are subject to manipulation and isolation from the rest of the mechanism" ((Glennan 2008, 378), quoted in (Kuhlmann and Glennan 2014)) but many mechanist accounts dispense from such a strict requirement of modularity (e.g. (MDC), (Bechtel 2009) and (Illari and Williamson 2012)).

Finally, a notable case study was recently displayed in the debate over the physical status of the Higgs phenomenon, often called the Higgs 'mechanism', through which particles gain mass in the Standard Model. In the late 1950s the promising hypothesis was investigated that protons and neutrons gained mass through the spontaneous breaking of some symmetry. However, under such a hypothesis the theory seemed to predict the appearance of massless bosons (the Goldstone bosons), which evidence strongly suggests do not exist and that were therefore considered unphysical. During 1960s, several independent researchers (Peter Higgs, after which the phenomenon was named, was between them) formulated a theoretical argument blocking the appearance of Goldstone bosons. The first step of such an argument consists in the introduction of additional fields, thanks to which the symmetry group of the Lagrangian becomes gauge invariant. Secondly, with a suitable choice of gauge one can obtain a unitary gauge where the Goldstone boson disappears and the resulting field possesses mass. ${ }^{11}$ This merely formal account is often illustrated through a metaphor according to which the Gauge boson "has eaten the Goldstone boson and grown heavy" (Coleman 1985, p. 123). However, many philosophers of science have been critical about this semi-popular explanation of the reality behind the phenomenon. Margaret Morrison (2003), for instance, argues that the Higgs mechanism, as Maxwell's aether, possesses great heuristic importance, but is not realistically interpretable. According to Earman "[r]eaders of Scientific American can be satisfied with these justso stories. But philosophers of science should not be. For a genuine property like mass cannot be gained by eating descriptive fluff, which is just what gauge is." (Earman 2004, 1239). Lyre (2012) argues that, despite its name, the Higgs mechanism does not provide a causal dynamical story necessary to ground a ME, while Stolzner (2016) relates the explanatory import of the formal account to its unifying power, rather than to its mechanical underpinning.

\section{The Role of Mechanistic Explanation in Physics}

In the previous section, we have shown how some explanations in physics are incompatible with the mechanistic account. It might be tempting to conclude that a law-based account of explanation is to be favoured in this domain of science. After all, the argument would go, the familiar

\footnotetext{
${ }^{11}$ For an historical account of the discovery see (Baggott 2012). See (Coleman 1985) for a technical introduction.
} 
illustrative examples cited in section 2 are translatable in the form of derivations from laws.

Contra such a conclusion, in this section we want to show that, in physics as well as in special sciences, a comprehensive account of scientific explanation and of its role in scientific practice requires the appeal to the notion of ME.

One way of cashing out the contribution of a variety of explanation in science is the analysis of its role as a heuristic guide. MEs are guides to theory-testing, due to the ways in which they suggest manipulations, and to discovery, since "if one knows what kind of activity is needed to do something, then one seeks kinds of entities that can do it, and vice versa" (MDC, 17). On the contrary, an account that reduces explanation to the articulation of laws covering the explanandum does not display the same richness when applied to scientific practice (Bechtel and Abrahamsen 2005). Under this perspective, attempts at explaining phenomena by showing how things work has traced the path of scientific change throughout the history of physics. As an example, Maxwell's mechanical model of electromagnetic waves has been of crucial heuristic importance for the discovery of the electromagnetic nature of light (Nersessian 1984).

Another way of demonstrating the role of ME in physics is through the analysis of cases where ME reflects and clarifies the desiderata of a suitable explanation, in a far richer way than law-based theories of explanation. As an illustrating example of how the mechanistic account can be superior to law-based accounts of explanation, in the rest of this section we analyse a time-honoured problem in quantum theory: the measurement problem.

We can summarize the measurement problem as the problem of accounting for the apparent determinateness of results in quantum measurement. ${ }^{12} \mathrm{~A}$ widespread approach to this problem is the so-called 'black box' approach - that, rather than providing a genuine explanation, takes the notion of measurement as primitive and provides instructions for obtaining probabilities via the Born rule. The evolution of the quantum state in case of measurement is here described by the projection postulate, although it is typically unclear whether the latter should be interpreted as a physical process, or as a change in our epistemic state. ${ }^{13}$

Part of the attractiveness of the black box approach consists in its avoidance of commitments over the kinds of entities and processes that constitute the world. However, without a description of measurement interactions, traditional black box solutions fail to provide a clear-cut criterion for the application of the projection postulate. This, in turn, complicates the possibility of a solution to the many issues gravitating around the measurement problem. For instance, depending on whether or not the projection postulate is interpreted as a real physical process (the so-called 'collapse of the wave function'), the theory has different implications with respect to related problems of internal and

12 See section 4 of this volume for more details.

13 See Maroney (this volume) for details on this dispute. 
external consistency (e.g. Wigner's friend scenarios (Wigner 1995 (1961)), or non-locality and the relationship with special relativity).

In this sense, the standard black box solution does not provide a satisfactory explanation to the measurement problem - and yet, this limit seems not to be highlighted within the covering law (Hempel 2001, 281) or the unificationist models (Friedman 1974, 18). Contrarily to law-based accounts of explanation, and as we are about to argue more in details, the mechanistic account shows why, far from being motivated uniquely by metaphysical (as opposed to scientific) interests, so-called 'interpretative' claims have a substantial role in a satisfactory explanation of the determinateness of our experience.

Recently, other accounts have adopted a refined black box approach. In particular, antirealist interpretations of quantum theory (e.g. Fuchs et al. 2014) put forward an explanation away of the measurement problem where the determinateness of our experiences is seen as a fundamental but unproblematic brute fact. In such epistemic interpretations, the quantum state does not represent the outside world, but rather our mental state. For instance, according to QBism "quantum mechanics is a tool anyone can use to evaluate, on the basis of one's past experience, one's probabilistic expectations for one's subsequent experience" (Fuchs et al. 2014, p.1).

If the quantum state only represents our epistemic state, then the question 'why do we have determinate experiences?' does not araise in the first place: why should we expect, in a theory about mental states, anything else than determinate experiences?

Felline (forthcoming) argues that this kind of antirealist approaches represents a Pyrrhic victory rather than a genuine solution to the measurement problem, since they are achieved at the price of dragging quantum theory outside the domain of empirical sciences. One of the pernicious consequences of this move is that explanations in quantum theory do not submit to the same standards of scientificity (e.g. that explanatory claims imply successful empirical predictions) as the rest of empirical science.

In any case, this kind of antirealist solutions to the measurement problem rejects a necessary precondition of the inquiry carried in this chapter, i.e. that physics, as, more in general, empirical science, can represent the outside world. In as such, they are outside the scope of this chapter. In the following, we assume that a realist interpretation of quantum theory, according to which the theory describes the outside world, is possible.

Contrary to black-box approaches, the majority of proposals deems necessary, for a comprehensive and coherent quantum theory, the explanation of the determinateness of measurement results. In turn, such an explanation requires the opening of the black box, i.e. the description of the interactions that characterize measurement processes and the clear interpretation of the projection postulate. In being guided by the 'opening the black box' prescription, the proposals go beyond a law- 
based view of scientific explanation and are driven instead by the same spirit that we consider at the core of ME and summarize with the expression 'showing how things work'. In this sense, the development of the debate over the measurement problem is driven by the search for a ME.

This being said, specific solutions come in a variety of proposals that require further analysis.

An important divide among the explanations provided by different interpretations of quantum theory concerns the role played by the wave-function, an entity defined in $3 N$-dimensional configuration space, where $N$ is the number of particles considered.

In the most straightforward reading of the quantum formalism, the wave-function is a concrete entity inhabiting configuration space.

"The sorts of physical objects that wave-functions are, on this way of thinking, are (plainly) fields - which is to say that they are the sorts of objects whose states one specifies by specifying the values of some set of numbers at every point in the space where they live" (Albert 1996, 278)

GRW theory (Ghirardi, Rimini, Weber 1986, see also Lewis (this volume)), Everettian interpretations (Saunders (this volume)) and Bohmian mechanics (see Tumulka (this volume)) are often interpreted in this way.

What kind of explanation is at stake in these theories? Can a description of the measurement process where the wave-function plays a constitutive role, ground a ME? Yes, if we adopt - as we think we should - a mechanistic account that does not rule out a priori entities that do not live on a three-dimensional space. The above-cited theories describe different mechanisms underlying the occurrence of determinate measurement results. GRW implements a stochastic dynamics, according to which the wave-function randomly collapses into one of the terms of the superposition.

According to Everettian interpretations, the wave-function does not collapse, but all branches of the superposition coexist after a measurement and all possible results are realized. Here, decoherence shows how classical behaviour (i.e. the loss of interference between branches) is caused to emerge from dynamical processes described by Schrödinger equation.

Finally, in Bohmian mechanics the wave-function is interpreted as acting on one 'universal particle' like a physical field in configuration space (Albert 1996, 278). In this mechanistic decomposition, the interaction between wave-function and universal particle constitutes a new fundamental interaction.

It might be objected at this point that the acknowledgement of the wave function as a mechanistic entity goes far beyond our most liberal pre-theoretical image of mechanism. Indeed, many accounts (e.g. Craver 2007) describe mechanisms as systems in space-time. However, revolutionary extensions of the stable set of mechanistic elements are not only allowed, but also envisaged by the new mechanistic account, as the above-cited example of the discovery of the electromagnetic field clearly illustrates. Before Maxwell, only material bodies were said to causally 
interact (by contact or at a distance) between each other. Maxwell's realist interpretation of the electromagnetic field forced the abandonment of this assumption and added the interaction between material bodies and physical space to the basic elements of a ME. In the same way, within wavefunction realist interpretations of QT the wave-function is causally active, and should therefore be accepted as a basic element of a ME.

Besides being compatible with the explanations provided by wave-function ontologies, and contrarily to law-based accounts of explanation, the mechanical account is highly informative about the role that explanation has in the foundations of quantum theory.

For instance, an explanatory deficiency of $3 N$-dimensional wave-function ontologies is that it is not clear how they cover the manifest image of everyday 3-dimensional objects with their dynamics, from a $3 N$-dimensional object. If, in other words, the world is in $3 N$-dimensions, how then do you get our 3-dimensional world?

"the concern with these theories is that, because the wave-function lives on configuration space and not three-dimensional space, the explanatory scheme developed in classical theories in terms of a primitive ontology must be drastically revised. A new explanatory scheme is needed, and nobody has found one yet." (Allori 2013, 13. Page numbering refers to the online version)

The new mechanistic philosophy sheds a clarifying light over the issue raised in the above quote: the relation that typically grounds the relationship of explanatory relevance between higher and lower mechanistic levels is composition; however, there is no straightforward compositional relation between the (higher level) three-dimensional entities of our experience and the (lower level) wave-function in $3 N$ dimensions.

In Bohmian mechanics, a way to overcome this problem is "to introduce the notion of a multifield, a configuration of which assigns properties to sets or fusions of $n$ points, and to view n-particle wave-functions as corresponding to multi-fields on ordinary three-space rather than to fields on the much larger configuration space of the system." (Belot, 2012, 5-6) In this interpretation, 3dimensional macroscopic objects are composed of 3-dimensional microscopic objects, and this relationship makes it possible for the lower level mechanism to explain the behaviour of the higher level entities. Another notable solution, this time in the context of Everettian decoherence-based explanations of determinateness, is put forward in (Wallace 2003, 2010) - a strategy pivoting on a functionalist criterion for the identification of macro-objects, in concordance with the perspectivalist account of mechanistic description and explanation (e.g. Craver 2013).

The direct rival of the wave-function ontology is the so-called primitive ontology approach (Allori et al. 2008), based on the assumption that the wave-function has a 'nomological status', while the only real physical entities are three-dimensional objects with a well-defined dynamics. Under this approach, spontaneous collapse interpretations can be articulated with an ontology of flashes (Bell 
1987) or density of stuff (Benatti et al. 1985). Many-worlds theories might be implemented with a similar 3-dimensional density field ontology (Allori et al. 2011); however, the bulk of the work in this direction was developed in the framework of Bohmian mechanics.

In the version originally put forward in (Dürr et al. 1992), the primitive ontology approach can't be covered by the mechanistic account. In fact, while in the latter only concrete objects of physical reality, their properties and organization play an explanatory role, in this interpretation of Bohmian mechanics the wave-function is not part of the basic ontology of the world, and yet it plays an irreplaceable explanatory role. Following this view, Goldstein and Zanghì (2013) describe the wavefunction as a 'nomological entity' that 'governs' the behaviour of particles (going in this way beyond the ontologically neutral view of laws as exceptionless regularities). However, if we discard a naive literal reading of the 'governing' talk, it is unclear how this metaphor should be interpreted. Notice that, since the explanatory role of the wave-function as a nomological entity goes beyond the epistemic, formal role that laws as exceptionless regularities hold in the covering law (or the unificationist) model of explanation, this version of the primitive ontology approach does not naturally fit with law-based accounts of explanation, either. It is because of the unclear explanatory import of this version of the primitive ontology approach that, notwithstanding the apparent incompatibility of such an account with ME, its use as a counterexample to the ME account begs the question.

Moreover, other primitive ontologies exist that provide alternatives to the 'governing' explanation, easily accountable as mechanistic, as for instance dispositionalist interpretations (e.g. Esfeld et al. 2013), that avoid any appeal to laws of nature, or Humean approaches (e.g. Callender 2015), where laws of nature play a role that is openly conceded by the new mechanistic philosophy (Craver and Kaiser 2013).

This short survey of solutions to the measurement problem shows that the mechanistic account is not only compatible with (some explanations provided by) contemporary approaches to quantum theory, but it is also highly informative with respect to the constraints and desiderata of a satisfactory solution to the measurement problem, and to the role that explanation has in the foundations of quantum theory.

To this conclusion, it might be countered that the measurement problem, together with the other issues belonging to the so-called 'interpretation' of quantum theory, is a metaphysical, rather than a scientific, problem. However, such a categorical distinction between science and metaphysics is inadequate when applied to issues like the measurement problem, which, pivotal as it is for the achievement of a coherent quantum theory, can't be considered external to science. On the contrary, this survey illustrates how the new mechanistic account of explanation has the virtue of showing that and how the question of what kind of entities and processes underlie phenomena is indeed part and 
parcel of physics and science.

\section{Acknowledgment}

For suggestions and comments, I am very grateful to: Valia Allori, Michael Cuffaro, Mauro Dorato, Meinard Kuhlmann, Matteo Morganti, Davide Romano, Emanuele Rossanese, Alastair Wilson.

\section{Bibliography}

Albert, D. Z. (1996). "Elementary Quantum Metaphysics." In: J. Cushing, A. Fine, and S. Goldstein (eds.), Bohmian Mechanics and Quantum Theory: An Appraisal. Boston Studies in the Philosophy of Science, 184: 277-284.

Allori, V. (2013). "Primitive ontology and the structure of fundamental physical theories". In: Ney, A., \& Albert, D. Z. (Eds.). (2013). The wave function: Essays on the metaphysics of quantum mechanics. Oxford University Press, 58-75. Page refs. http://philsciarchive.pitt.edu/9342/1/AlloriWFOLast.pdf

Bechtel, W. (2009). "Explanation: Mechanism, modularity, and situated cognition.” Robbins, P., \& Aydede, M. (Eds.). The Cambridge handbook of situated cognition. Cambridge: Cambridge University Press, 155-170.

Bechtel W. and A. Abrahamsen (2005). "Explanation: A mechanist alternative." Studies in History and Philosophy of Science Part C: Studies in History and Philosophy of Biological and Biomedical Sciences, 36(2), 421-441.

Bechtel, W. and R.C. Richardson (2010) [1993]. Discovering Complexity: Decomposition and Localization as Strategies in Scientific Research, Second Edition. Cambridge, MA: MIT Press/Bradford Books.

Baggott, J. (2012). Higgs: The Invention and Discovery o the 'God Particle'. Oxford: Oxford University Press.

Bell, J. S. (1987). Speakable and Unspeakable in Quantum Mechanics. Cambridge: Cambridge University Press.

Benatti, F., G. C. Ghirardi, and R. Grassi (1995). "Describing the Macroscopic World: Closing the Circle within the Dynamical Reduction Program”. Found. Phys., 25: 5 - 38.

Callender, C. (2015). “One world, one beable”. Synthese, 192(10), 3153-3177

Coleman, S. (1985) Aspects of Symmetry: Selected Erice Lectures. Cambridge: Cambridge University Press.

Craver, C. F. (2007). Explaining the brain. Oxford: Oxford University Press.

Craver, C. F. (2013). Functions and mechanisms: A perspectivalist view. In Functions: Selection and 
mechanisms (pp. 133-158). Springer, Dordrecht.

Craver, C. F., \& Kaiser, M. I. (2013). Mechanisms and laws: Clarifying the debate. In Mechanism and causality in biology and economics (pp. 125-145). Springer, Dordrecht.

Dorato, M., and Felline, L. (2011). "Scientific explanation and scientific structuralism". In A. Bokulich and P. Bokulich (Eds.), Scientific structuralism (pp. 161-176). Dordrecht: Springer.

Dürr, D., S. Goldstein, and N. Zanghì (1992). "Quantum Equilibrium and the Origin of Absolute Uncertainty". Journal of Statistical Physics. 67, 843-907.

Earman, J. (2004). "Laws, symmetry, and symmetry breaking: Invariance, conservation principles, and objectivity". Philosophy of Science 71, 1227-1241.

Esfeld, M., Hubert, M., Lazarovici, D., and Dürr, D. (2013). "The ontology of Bohmian mechanics". The British Journal for the Philosophy of Science, axt019.

Felline, L. (2015). "Mechanisms meet structural explanation”. Synthese, First online: 30 May: 1-16.

Felline, L. (2016). "It's a Matter of Principle: Scientific Explanation in Information-Theoretic Reconstructions of Quantum Theory". Dialectica, 70 (4): 549-575.

Felline, L. (forthcoming). "Quantum Theory is not only about Information" Studies in History and Philosophy of Modern Physics.

Friedman, M. (1974). "Explanation and scientific understanding”. The Journal of Philosophy, 71(1), 5-19.

Fuchs, C. A., Mermin, N. D., \& Schack, R. (2014). “An introduction to QBism with an application to the locality of quantum mechanics". American Journal of Physics, 82(8), 749-754. https://arxiv.org/pdf/1311.5253.pdf.

Glennan, S. S. (2002). "Rethinking Mechanistic Explanation.” Philosophy of Science 69(S): S342S353.

Glennan, S. S. (2008). "Mechanisms". In M. Curd \& S. Psillos (Eds.), Routledge companion to the philosophy of science (pp. 376-384). New York: Routledge.

Glennan, S. S. (2011). "Singular and General Causal Relations: A Mechanist Perspective". In Causality in the Sciences, in McKay Illari, P., Russo, F., and J. Williamson (eds.): Causality in the Sciences, Oxford: Oxford University Press, 89-817.

Glennan, S. (2017). The new mechanical philosophy. Oxford University Press.

Goldstein, S., \& Zanghì, N. (2013). "Reality and the role of the wave function in quantum theory". In: Ney, A., \& Albert, D. Z. (Eds.). The wave function: Essays on the metaphysics of quantum mechanics. Oxford University Press, 91-109.

Hall, N. (2004). “Two concepts of causation”. In Causation and counterfactuals, Collins, J. D., Hall, E. J., \& Paul, L. A. (eds.). MIT Press, 225-276.

Hedström, P., \& Swedberg, R. (1998). "Social Mechanisms: An Introductory Essay." In Social 
Mechanisms: An Analytical Approach to Social Theory, edited by Peter Hedström and Richard Swedberg, Cambridge, U.K. ; New York: Cambridge University Press, 1-31.

Hempel, C. G. (2001). The philosophy of Carl G. Hempel: studies in science, explanation, and rationality. Oxford University Press.

Hesse, M. B. (2005) [1961]. Forces and fields: The concept of action at a distance in the history of physics. Courier Corporation.

Illari, P. M., \& Williamson, J. (2012). "What is a mechanism? Thinking about mechanisms across the sciences". European Journal for Philosophy of Science, 2(1), 119-135.

Kochiras, H. (2013). “The Mechanical Philosophy and Newton's Mechanical Force”. Philosophy of Science, 80(4), 557-578.

Kuhlmann, M. (2011). "Mechanisms in dynamically complex systems," in McKay Illari, P., Russo, F., and J. Williamson (eds.): Causality in the Sciences, Oxford: Oxford University Press, 880 906.

Kuhlmann, M., \& Glennan, S. (2014). "On the relation between quantum mechanical and neomechanistic ontologies and explanatory strategies." European Journal for Philosophy of Science, 4(3), 337-359.

Kuhlmann, M. (2017). "Mechanisms in Physics", Kuhlmann, M. (2017). Mechanisms in physics. Glennan, Stuart und Phyllis Illari (eds): Routledge Handbook of the Philosophy of Mechanisms.

Lange, M. (2011). “Conservation laws in scientific explanations: Constraints or coincidences?”. Philosophy of Science, 78(3), 333-352.

Lyre, H. (2012). “The just-so Higgs story: A response to Adrian Wüthrich”. Journal for general philosophy of science, 43(2), 289-294.

Machamer, P., Darden, L., \& Craver, C. F. (2000). “Thinking about mechanisms". Philosophy of science, 67(1), 1-25.

Morrison, M. (2003). "Spontaneous symmetry breaking: Theoretical arguments and philosophical problems". Symmetries in physics: Philosophical reflections, 347-363.

Nersessian, N. J. (1984). "Aether/or: The creation of scientific concepts". Studies in History and Philosophy of Science Part A, 15(3), 175-212.

Salmon, W. C. (1984). Scientific Explanation and the Causal Structure of the World, Princeton University Press.

Stöltzner, M. (2016). The variety of explanations in the Higgs sector. Synthese, 1-28.

Creath, R. "Logical Empiricism", The Stanford Encyclopedia of Philosophy (Fall 2017

Edition), Edward N. Zalta (ed.), URL =
$<$ https://plato.stanford.edu/archives/fall2017/entries/logical-empiricism/>. 
Wallace, D. (2003). "Everett and Structure." Studies in History and Philosophy of Modern Physics 34, 87-105.

Wallace, D. (2010). "Decoherence and Ontology." In Simon Saunders, Jonathan Barrett, Adrian Kent, and David Wallace, eds., Many Worlds?: Everett, Quantum Theory, and Reality, pp. 53-72. Oxford: Oxford University Press.

Wigner, E. P. (1995). "Remarks on the mind-body question". In Philosophical Reflections and Syntheses, pp. 247-260. Springer, Berlin, Heidelberg. First published in Wigner, E. P. (1961) "The Scientist Speculates". Heinmann, London. 